\title{
On the interaction of massive spinor particles with external electromagnetic and torsion fields
}

\author{
Lewis H. Ryder ${ }^{a}$ 日 and Ilya L. Shapiro ${ }^{b, c}$ Z \\ a Physics Laboratory, University of Kent, Canterbury, Kent, CT2 7NR, UK \\ b Departamento de Fisica, Universidade Federal de Juiz de Fora, 36036-330, MG - Brazil \\ c Tomsk State Pedagogical University, 634041, Tomsk, Russia
}

Abstract. We explore the Dirac equation in external electromagnetic and torsion fields. Motivated by the previous study of quantum field theory in an external torsion field we include a nonminimal interaction of the spinor field with torsion. As a consequence the torsion axial vector and the electromagnetic potential enter the action in a similar form. The existence of an extra local symmetry is emphasized and the FoldyWouthuysen transformation is performed to an accuracy of next to the leading order. We also discuss the motion of a classical test particle in a constant torsion field.

Introduction. Research in the field of gravity with torsion and especially the interaction of torsion with a spinor field has attracted attention for a long time [1, 2, 3, 4, 5] (see [7] for a recent review of gravity with torsion). Recently interest in theories with torsion has increased because of the success of the formal development of string theory [6] which is (together with its modifications and generalizations) nowadays regarded as the main candidate for the unique description of all quantum fields. String theory predicts the existence of the space-time torsion. Probably this was the reason why in recent years there has been an increasing interest in possible physical effects of torsion [8, 9, 10, 11, 12]. Most of these works discuss the effects of classical or quantum matter fields on an external torsion background.

The study of the renormalization of quantum field theory in an external gravitational field with torsion 13, 14 has shown that the interaction of matter fields with torsion has special features. If the theory includes Yukawa interaction, then the renormalizability requires nonminimal interaction of torsion with both spinors and scalars. Thus we arrive at the necessity to introduce some new nonminimal parameters which characterizes such an interaction. The considerations of [8, 9, 11, 12] were based on the nonrelativistic limit of the Dirac equation - that is on the generalization of the Pauli equation for the case of an external electromagnetic and torsion fields. The main purpose of the present paper is to derive the next to the leading order corrections to the Pauli equation in the framework of the Foldy-Wouthuysen transformation. We also demonstrate some global symmetry which holds for the Dirac spinor in external electromagnetic and torsion fields.

The paper is organized in the following way. In the next section we introduce basic notations for the gravity with torsion. Some additional symmetry which holds for the spinor field coupled to torsion is established. In section 3 the details of the Foldy-Wouthuysen transformation are presented. The equations of motion for spinning particles are discussed in section 4 and section 5 contains our conclusions.

2. Quantum fields in an external gravitational field with torsion. Let us start with the basic notions of gravity with torsion. All our notations correspond to those in [14]. The metric $g_{\mu \nu}$ and torsion $T_{\beta \gamma}^{\alpha}$ are independent characteristics of space - time. When torsion is present, the

\footnotetext{
${ }^{1}$ e-mail address: 1.h.ryder@uk.ac.uk

2 e-mail address: shapiro@ibitipoca.fisica.ufjf.br
} 
covariant derivative $\tilde{\nabla}$ is based on the nonsymmetric connection $\tilde{\Gamma}_{\beta \gamma}^{\alpha}$

$$
\tilde{\Gamma}_{\beta \gamma}^{\alpha}-\tilde{\Gamma}_{\gamma \beta}^{\alpha}=T_{\beta \gamma}^{\alpha}
$$

The metricity condition $\tilde{\nabla}_{\mu} g_{\alpha \beta}=0$ enables one to express the connection through the metric and torsion in a unique way [14].

It is convenient to divide the torsion field into three irreducible components: i) the vector $T_{\beta}=T_{\beta \alpha}^{\alpha}$, ii) the axial vector $S^{\nu}=\epsilon^{\alpha \beta \mu \nu} T_{\alpha \beta \mu}$ and iii) the tensor $q_{\beta \gamma}^{\alpha}$, which satisfies conditions $q_{\beta \alpha}^{\alpha}=0 ; \epsilon^{\alpha \beta \mu \nu} q_{\alpha \beta \mu}=0$. The torsion field can be always expressed through these new fields as

$$
T_{\alpha \beta \mu}=\frac{1}{3}\left(T_{\beta} g_{\alpha \mu}-T_{\mu} g_{\alpha \beta}\right)-\frac{1}{6} \varepsilon_{\alpha \beta \mu \nu} S^{\nu}+q_{\alpha \beta \mu}
$$

We remark that the string effective action contains the completely antisymmetric torsion which can be described by the axial vector $S_{\alpha}$.

To construct the actions of the matter fields in an external gravitational field with torsion one imposes the principles of locality, and general covariance and require the symmetries of the given theory (like gauge invariance for the QED or SM) in flat space-time to hold for the generalized theory in curved space-time with torsion, and forbid the introduction of the new parameters with the dimension of inverse mass. It turns out that these demands fix the form of the action apart from the values of some new parameters which remain arbitrary. These new quantities are called nonminimal parameters and parameters of the vacuum action.

Along with the nonminimal scheme described above, there is a (more traditional) minimal one. According to it the partial derivatives $\partial_{\mu}$ are substituted by the covariant ones $\tilde{\nabla}_{\mu}$, the flat metric $\eta^{\mu \nu}$ by $g^{\mu \nu}$ and the volume element $d^{4} x$ by the covariant expression $d^{4} x \sqrt{-g}$. For the Dirac spinor this procedure leads to the expression (see, for example, [2, 14])

$$
S_{\frac{1}{2}, \min }=i \int d^{4} x \sqrt{-g} \bar{\psi}\left(\gamma^{\alpha} \partial_{\alpha}+i \eta_{1} \gamma_{5} \gamma^{\alpha} S_{\alpha}-i \eta_{2} \gamma^{\alpha} T_{\alpha}-i m\right) \psi
$$

with $\eta_{1}=-1 / 8$ and $\eta_{2}=0$. Therefore minimally the Dirac spinor interacts only with the $S_{\alpha}$ part of the torsion tensor (2).

The nonminimal interaction is essentially more complicated. In the spinor sector the nonminimal interaction is given by (3) with an arbitrary $\eta_{1,2}$. The introduction of the nonminimal parameters in the matter field sector of GUT and also the vacuum action is dictated by the requirements of renormalizability, because corresponding counterterms appear even if the starting theory had minimal interactions only [13]. One has to remember, however, that among all the nonminimal parameters only the ones which have relation to the axial piece $S_{\mu}$ of the torsion tensor are essential [13], 14]. Since we are only interested in the torsion effects, in the rest of this paper we will keep the metric flat.

In this letter we will be concerned by the action of the nonminimally coupled Dirac spinor in external electromagnetic and torsion fields (from this point we consider a flat metric only). We notice, that since the trace of torsion $T_{\mu}$ and the electromagnetic field $A_{\mu}$ appear only in the combination $e A_{\mu}+\eta T_{\mu}$, it is natural to denote this combination simply $e A_{\mu}$ and therefore we can drop $T_{\mu}$ everywhere. Moreover we change $\eta_{1}$ to $\eta$.

$$
S_{1 / 2}=i \int d^{4} x \bar{\psi}\left[\gamma^{\alpha}\left(\partial_{\alpha}-i e A_{\alpha}+i \eta \gamma_{5} S_{\alpha}\right)-i m\right] \psi
$$

It is well known that for the spinor field in an external electromagnetic field the gauge transformation leaves the action invariant. In the case of a massless action (4) this transformation can be generalized in the following way:

$$
\psi \rightarrow \psi^{\prime}=\psi e^{\alpha+\beta \gamma_{5}}, \quad \bar{\psi} \rightarrow \bar{\psi}^{\prime}=\bar{\psi} e^{-\alpha+\beta \gamma_{5}},
$$




$$
A_{\mu} \rightarrow A_{\mu}^{\prime}=A_{\mu}-\frac{1}{e} \partial_{\mu} \alpha, \quad S_{\mu} \rightarrow S_{\mu}^{\prime}=S_{\mu}-\frac{1}{\eta} \partial_{\mu} \beta
$$

where $\alpha=\alpha(x)$ and $\beta=\beta(x)$ are scalar and pseudoscalar parameters of transformation. Despite this symmetry is softly broken by the mass of the spinor field in (4), it turns out to be very useful. In particular, if the torsion field is considered as an external background, this extra symmetry explains the special "gauge invariant" form of divergences of the effective action of torsion which was observed in [15, 14] for the case of massless spinor field.

If we intend to consider the propagating torsion, such a symmetry puts rigid restrictions on the form of the action for the $S_{\mu}$ field [16]. The torsion field is supposed to interact with both massive and massless spinors. Then one can show that the only possible form of the torsion action

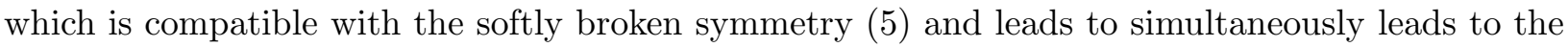
renormalizable quantum theory is

$$
S_{t s}=\int d^{4} x\left[-\frac{1}{4} S_{\mu \nu}^{2}+\frac{1}{2} M_{t s}^{2} S_{\mu}^{2}\right]+(\text { interaction terms })
$$

where $S_{\mu \nu}=\partial_{\mu} S_{\nu}-\partial_{\nu} S_{\mu}$ and $M_{t s}$ is the mass of the torsion field.

Thus the only one propagating part of the torsion axial vector is the transversal one, while the appearance of the longitudinal part of $S_{\mu}$ is due to the massive terms in (6) and (4), and the equation of motion for this part of $S_{\mu}$ is algebraic with the corresponding part of spin tensor as a source. Indeed this doesn't put any restrictions on the value of this part which can be nonzero and even large in a very early Universe. On the other hand, even if the longitudinal component of torsion could be large at the moments close to the Big Bang; during the inflationary period it could decrease in many orders of magnitude [15] and moreover the inhomogeneities of such a primeval torsion could become irrelevant even at the cosmic distances. Therefore, despite the propagation of torsion is related to the transversal part of $S_{\mu}$, there is nothing inconsistent to suppose that the primeval torsion has a nonzero longitudinal component. In particular, it can be constant field of an arbitrary (including the spacelike) configuration.

This supposition is in fact important for the discussion of the results of recent paper [10]. The main hypotheses of [10] is that there is a torsion background radiation $S_{\mu}=\left(S_{0}, \mathbf{S}\right)$ with a very small but nonzero constant $\mathbf{S}$. Then integrating over the spinor fields in the theory with an action (4) the Lagrangian of external fields $A_{\mu}, S_{\mu}$ results in the form $\epsilon^{\mu \nu \alpha \beta} A_{\mu} S_{\nu} F_{\alpha \beta}$. This Lagrangian is exactly the one which is necessary to explain the observed anisotropy of the cosmological electromagnetic propagation [17]. As one can easily see, the above Lagrangian is gauge invariant (with respect to the usual transformation of the electromagnetic potential $A_{\mu}$ ) if only $S_{\mu}$ is a longitudinal axial vector. Therefore the existence of this component is a necessary constituent of the mentioned explanation.

Another form of the torsion field arises if we suppose that the torsion tensor doesn't violate the isotropy of the Universe. Then in our cosmological frame the time component of the vector $S_{\mu}$ should dominate. The existing inflationary cosmological model with torsion predicts that during the period of inflation the torsion field decreased exponentially 15 . On the other hand during this period all possible inhomogeneities of torsion have to disappear since we observe some small part of the Universe. Therefore today we would observe only a very weak and weakly dependent in space and time components of the axial vector $S_{\mu}$.

The question whether the torsion field really exists can be solved only experimentally, so it is important to study the features of different torsion configurations. Thus it is worth of studying the possible manifestations of torsion at low energies, where there is a possibility of making very precise measurements.

3. Foldy-Wouthuysen transformation. In this section we shall derive the Foldy-Wouthuysen transformation for the Dirac spinor in external torsion and electromagnetic fields. The initial 
Hamiltonian corresponding to (画), has the form:

$$
H=\beta m+\mathcal{E}+\mathcal{G}
$$

where $\mathcal{E}=e A_{0}-\eta \gamma_{5} \alpha \mathbf{S}, \quad \mathcal{G}=\alpha(\mathbf{p}-e \mathbf{A})+\eta \gamma_{5} S_{0}$ are the even and odd parts of the expression, $S_{\mu}=\left(S_{0}, \mathbf{S}\right)$ is the axial piece of the torsion tensor (2) and we have used the standard representation of the gamma-matrices and the units $c=\hbar=1$.

Our purpose is to find a unitary transformation which separates "small" and "large" components of the Dirac spinor. We use a general prescription

$$
H^{\prime}=e^{i \mathcal{S}}\left(H-i \partial_{t}\right) e^{-i \mathcal{S}}
$$

where $\mathcal{S}$ has to be chosen in an appropriate way. We shall find $\mathcal{S}$ and $H^{\prime}$ in a form of the nonrelativistic expansion, and thus will take $\mathcal{S}$ to be of order $1 / \mathrm{m}$. Then we can use standard result (see, for example, [18])

$$
\begin{aligned}
H^{\prime}=H+i[\mathcal{S}, H] & -\frac{1}{2}[\mathcal{S},[\mathcal{S}, H]]-\frac{i}{6}[\mathcal{S},[\mathcal{S},[\mathcal{S}, H]]]+\frac{1}{24}[\mathcal{S},[\mathcal{S},[\mathcal{S},[\mathcal{S}, H]]]]- \\
& -\dot{\mathcal{S}}-\frac{i}{2}[\mathcal{S}, \dot{\mathcal{S}}]+\frac{1}{6}[\mathcal{S},[\mathcal{S}, \dot{\mathcal{S}}]]+\ldots
\end{aligned}
$$

One can easily see that $\mathcal{E}$ and $\mathcal{G}$ given above (anti)commute with $\beta$ in a standard way $\mathcal{E} \beta=$ $\beta \mathcal{E}, \mathcal{G} \beta=-\beta \mathcal{G}$ and therefore one can safely use the standard prescription for the lowest-order approximation for $\mathcal{S}: \mathcal{S}=-\frac{i}{2 m} \beta \mathcal{G}$.

$$
H^{\prime}=\beta m+\mathcal{E}^{\prime}+\mathcal{G}^{\prime}
$$

where $\mathcal{G}^{\prime}$ is of order $1 / m$, and one has to perform second Foldy-Wouthuysen transformation with $\mathcal{S}^{\prime}=-\frac{i}{2 m} \beta \mathcal{G}^{\prime}$. This leads to the

$$
H^{\prime \prime}=\beta m+\mathcal{E}^{\prime}+\mathcal{G}^{\prime \prime}
$$

with $\mathcal{G}^{\prime \prime} \approx 1 / m^{2}$; and then a third Foldy-Wouthuysen transformation with $\mathcal{S}^{\prime \prime}=-\frac{i}{2 m} \beta \mathcal{G}^{\prime \prime}$ removes odd operators in the given order of the nonrelativistic expansion, so that we finally obtain the usual result

$$
H^{\prime \prime \prime}=\beta\left(m+\frac{1}{2 m} \mathcal{G}^{2}-\frac{1}{8 m^{3}} \mathcal{G}^{4}\right)+\mathcal{E}-\frac{1}{8 m^{2}}[\mathcal{G},([\mathcal{G}, \mathcal{E}]+i \dot{\mathcal{G}})]
$$

Substituting here $\mathcal{E}$ and $\mathcal{G}$ after some algebra we arrive at the final form of the Hamiltonian

$$
\begin{aligned}
H^{\prime \prime \prime}= & \beta\left[m+\frac{1}{2 m}\left(\mathbf{p}-e \mathbf{A}+\eta S_{0} \sigma\right)^{2}-\frac{1}{8 m^{3}} \mathbf{p}^{4}\right]+e A_{0}-\eta(\sigma \cdot \mathbf{S})-\frac{e}{2 m} \sigma \cdot \mathbf{B}-\frac{\eta^{2}}{m} \beta S_{0}^{2}- \\
- & \frac{e}{8 m^{2}}[\operatorname{div} \mathbf{E}+i \sigma \cdot \operatorname{curl} \mathbf{E}+2 \sigma \cdot(\mathbf{E} \times \mathbf{p})]+\frac{\eta}{8 m^{2}}\left[-\sigma \cdot \nabla \dot{S}_{0}+\left\{p_{i},\left\{p^{i},(\sigma \cdot \mathbf{S})\right\}\right\}-\right. \\
& -2\{(\mathbf{T} \cdot \mathbf{p}),(\sigma \cdot \mathbf{p})]-2 \operatorname{curl} \mathbf{S} \cdot \mathbf{p}+2 i(\sigma \cdot \nabla)(\mathbf{S} \cdot \mathbf{p})+2 i(\nabla \mathbf{S})(\sigma \cdot \mathbf{p})\}
\end{aligned}
$$

where we use standard notation $\{A, B\}=A B+B A$. One can indeed proceed in this way and get separated Hamiltonian with any given accuracy in $1 / \mathrm{m}$. The first five terms of (13) reproduce the Pauli-like equation with torsion derived in [8] (see also [9]). The next term is the next-to-the-leading order nonrelativistic correction for the case of external electromagnetic field without torsion. The remaining terms are torsion-dependent corrections to the Pauli-like equation [8] (see also [9, 11]). 
In those terms we follow the same system of approximation as is standard for the electromagnetic case [18]; that is we keep terms (kinetic energy) ${ }^{3}$ and (kinetic energy) ${ }^{2} \cdot($ potential energy).

Further simplifications are possible if we are interested in relic cosmological torsion. In this case we have to keep only the constant components of the axial $S_{\mu}$. Then the effects of torsion will be: i) a small correction to the potential energy of the spinor field, (which sometimes looks just like a correction to the mass) and ii) the appearance of a new gauge-invariant interaction spin-momentum term in the Hamiltonian.

4. Motion of spinning particle on the constant torsion background. The above expression (13) is rather cumbersome and difficult to work with. However, one can make some general observations. In particular, at this order of approximation the corrections to the Pauli-like equations which are related to the constant part $S_{0}$ of the axial piece of torsion enter the Hamiltonian only via the expression $\pi=\mathbf{p}-e \mathbf{A}+\eta S_{0} \sigma$. Therefore, according to the result of 8 ] the second order correction does not modify the equation of motion for the classical spinning particle.

Consider now the motion of a spinning particle in the space with torsion but without any electromagnetic field. The physical degrees of freedom of the particle are its coordinate $\mathbf{x}$ and its spin $\sigma$. For the reasons explained in the Introduction we are mainly interested in the cases of constant axial $S_{\mu}=\left(S_{0}, \mathbf{S}\right)$. In this case the corresponding equations of [8] have the form:

$$
\begin{gathered}
\frac{d \mathbf{v}}{d t}=-\eta \mathbf{S}(\mathbf{v} \cdot \sigma)-\frac{\eta S_{0}}{c} \frac{d \sigma}{d t} \\
\frac{d \sigma}{d t}=+\frac{2 \eta}{\hbar}[\mathbf{S} \times \sigma]-\frac{2 \eta S_{0}}{\hbar c}[\mathbf{v} \times \sigma]
\end{gathered}
$$

Consider first the case suggested in [10], when $S_{0}=0$ so that only $\mathbf{S}$ is present. Since $\mathbf{S}=$ const, we can safely put $S_{1,2}=0$. The solution for spin can be easily found to be

$$
\sigma_{3}=\sigma_{30}=\text { const }, \quad \sigma_{1}=\rho \cos \left(\frac{2 \eta S_{3} t}{\hbar}\right), \quad \sigma_{2}=\rho \sin \left(\frac{2 \eta S_{3} t}{\hbar}\right)
$$

where $\rho=\sqrt{\sigma_{10}^{2}+\sigma_{20}^{2}}$. For the first two components of the velocity we have $v_{1}=v_{10}=$ const, $v_{2}=v_{20}=$ const, but the solution for $v_{3}$ turns out to be complicated. For $\sigma_{3}=0$ one finds

$$
v_{3}(t)=v_{30}-\frac{\hbar \rho v_{20}}{2 m}-\frac{\rho \hbar v_{10}}{2 m} \sin \left(\frac{2 \eta S_{3} t}{\hbar}\right)+\frac{\rho \hbar v_{20}}{2 m} \cos \left(\frac{2 \eta S_{3} t}{\hbar}\right)
$$

and for $\sigma_{3} \neq 0$ the solution is

$$
\begin{gathered}
v_{3}(t)=\left[v_{30}+\frac{\rho \hbar\left(\sigma_{3} v_{10} \hbar-2 m v_{20}\right)}{4 m^{2}+\hbar^{2} \sigma_{3}^{2}}\right] e^{-\frac{\eta S_{3} \sigma_{3}}{m} t}- \\
-\frac{\rho \hbar}{4 m^{2}+\hbar^{2} \sigma_{3}^{2}}\left[\left(\sigma_{3} v_{10} \hbar-2 m v_{20}\right) \cos \left(\frac{2 \eta S_{3} t}{\hbar}\right)+\left(\sigma_{3} v_{20} \hbar+2 m v_{10}\right) \sin \left(\frac{2 \eta S_{3} t}{\hbar}\right)\right]
\end{gathered}
$$

Physically such a solution means i) precession of the spin around the direction of $\mathbf{S}$ and ii) oscillation of the particle velocity in this same direction is accompanied (for $\sigma_{3} \neq 0$ ) by the exponential damping of the initial velocity in this direction. We remark that the value of the relic torsion field should be very weak so that a very precise experiments will be necessary to measure this (probably extremely slow) precession, oscillation and damping. 
Consider finally the other special case $\mathbf{S}=0$, which is the form of the torsion field motivated by isotropic cosmological models. Then the equations of motion have a form different from the ones occurring in a magnetic field

$$
\frac{d \mathbf{v}}{d t}=-\frac{\eta S_{0}}{c} \frac{d \sigma}{d t}=\frac{2 \eta^{2} S_{0}^{2}}{c \hbar}[\mathbf{v} \times \sigma]
$$

Despite the fact that those equations formally look like a nonlinear system of equations for six unknowns, they can be integrated immediately if we notice that the time variations of the variables do not affect the vector product. Hence the general solutions are

$$
\begin{gathered}
\mathbf{v}(t)=\mathbf{v}_{0}+\left[\mathbf{v}_{0} \times \sigma_{0}\right] \frac{2 \eta^{2} S_{0}^{2}}{c \hbar} t \\
\sigma(t)=\sigma_{0}-\left[\mathbf{v}_{0} \times \sigma_{0}\right] \frac{2 \eta S_{0}}{\hbar} t
\end{gathered}
$$

The first equation indicates that the motion of such a particle is a motion with constant acceleration. This is possible, in the presence of torsion, for electrically neutral particles with spin. A remark is in order here. The torsion field is supposed to act on the spin of particles but not on their angular momentum [2]. Therefore a motion like (20) will occur for individual electrons or other particles with spin as well as for macroscopic bodies with a nonzero overall spin orientation but it does not occur for the (charged or neutral) bodies with a random orientation of spins. We note that the possibility of the accelerating motion of the spinning particles in an external torsion field has been discusses in [12].

5. Conclusions. We have considered some aspects of the motion of Dirac particles nonminimally coupled to external torsion and electromagnetic fields. The usual electrodynamic gauge invariance becomes generalized to (5). This symmetry transformation has one extra pseudoscalar parameter compared with standard gauge invariance but in this extended form it holds only in the massless sector of the action.

Despite the fact that the Dirac equation with an additional torsion background is more complicated than it is in the purely electromagnetic case, it admits the Foldy-Wouthuysen transformation which separates the "large" and "small" components of the Dirac spinor field. The higher order corrections to the Pauli equation do not involve the constant timelike component $S_{0}$ of the torsion dual axial.

The motion of classical spinning particles in an external torsion field shows some original features if the torsion field has only a time component $S_{0}$. In this case the motion with constant acceleration is possible for an electrically neutral particle.

Acknowledgments. One of the authors (I.L.Sh.) is grateful to Departamento de Física, Universidade Federal de Juiz de Fora for warm hospitality. His work was supported in part by the $\mathrm{CNPq}$ (Brazil) and by Russian Foundation for Basic Research under project No.96-02-16017. 


\section{References}

[1] B.K. Datta, Nuovo Cim. 6B (1971) 1; 16.

[2] F.W. Hehl, Gen. Relat.Grav.4(1973)333;5(1974)491; F.W. Hehl, P. Heide, G.D. Kerlick and J.M. Nester, Rev. Mod. Phys.48 (1976) 3641.

[3] J. Audretsch, Phys.Rev. 24D (1981) 1470.

[4] H. Rumpf, Gen. Relat. Grav. 14 (1982) 773.

[5] H. Rumpf, Gen. Relat. Grav. 10 (1979) 509; 525; 647.

[6] M.B. Green, J.H. Schwarz and E. Witten, Superstring Theory. (Cambridge University Press, Cambridge, 1987).

[7] "On the gauge aspects of gravity", F. Gronwald, F. W. Hehl, GRQC-9602013, Talk given at International School of Cosmology and Gravitation: 14th Course: Quantum Gravity, Erice, Italy, 11-19 May 1995, gr-qc/9602013

[8] V.G. Bagrov, I.L. Buchbinder and I.L. Shapiro, Izv. VUZov, Fisica (in Russian. English translation: Sov.J.Phys.) 35,n3 (1992) 5 (see also at hep-th/9406122).

[9] R. Hammond, Phys.Lett. 184A (1994) 409; Phys.Rev. 52D (1995) 6918.

[10] A. Dobado and A. Maroto, Mod.Phys.Lett. A 12 (1997) 3003.

[11] C. Lammerzahl, Phys.Lett. 228A (1997) 223.

[12] P. Singh and L.H. Ryder, Class.Quant.Grav. 14 (1997) 3513.

[13] I.L. Buchbinder and I.L. Shapiro, Phys.Lett. 151B (1985) 263; Class. Quantum Grav. 7 (1990) 1197.

[14] I.L. Buchbinder, S.D. Odintsov and I.L. Shapiro, Effective Action in Quantum Gravity. (IOP Publishing - Bristol, 1992).

[15] I.L. Buchbinder, S.D. Odintsov and I.L. Shapiro, Phys.Lett. 162B (1985) 92.

[16] A.S. Belyaev and I. L. Shapiro, hep-ph/9712503, Phys.Lett.B, to be published.

[17] B. Nodland and J. Ralston, Phys.Rev.Lett., 78 (1997) 3043.

[18] J.M. Bjorken and S.D. Drell, Relativistic Quantum Mechanics. (McGraw-Hill Book Company NY, 1964). 